\title{
Percepción del aprendizaje, procedimientos de evaluación y uso de la tecnología PowerPoint en la formación universitaria de Medicina
}

\author{
Ramon Cladellas Pros (iD, Antoni Castelló Tarrida (iD \\ Universitat Autònoma de Barcelona (Spain) \\ ramon.cladellas@uab.es, toni.castello@uab.es
}

Received June, 2016

Accepted November, 2016

\section{Resumen}

Objeto: Verificar si la percepción del proceso de aprendizaje, según se oriente a la comprensión o al recuerdo, está asociada a los procedimientos de tecnología docente empleados y de evaluación del mismo.

Diseño/metodología/enfoque: A partir de una muestra de 315 registros sobre asignaturas obligatorias de segundo curso de Grado, obtenidos a partir de 63 estudiantes universitarios de Medicina, se plantea la hipótesis de que aquéllas que utilizan evaluación tipo test y diapositivas con elevada carga verbal están más orientadas al recuerdo que las que emplean evaluaciones de otro tipo.

Aportaciones y resultados: Los resultados muestran que las materias orientadas al recuerdo hacen un mayor uso de tecnología PowerPoint y, particularmente, de formatos verbales, un peor rendimiento académico y una menor percepción de aprendizaje.

Limitaciones: Próximos estudios deberían contemplar muestras más grandes de asignaturas que abarcara un mayor número de cursos académicos y distintas carreras. 
Originalidad / Valor añadido: Hasta ahora ningún estudio ha contemplado de manera conjunta la incidencia de la percepción del aprendizaje, los procedimientos de evaluación y el uso de la tecnología del PowerPoint.

Palabras clave: Procedimiento de evaluación, Comprensión, Recuerdo, Tecnología docente

Códigos JEL: I12

Title: Perception of learning, assessment procedures and the use of PowerPoint technology in University Studies of Medicine

\section{Abstract}

Purpose: Test whether how professors represent the learning process (understanding-oriented or recall-oriented) is connected to the evaluation procedures and to the use of teaching technology.

Design/methodology: 63 students produced 315 records referred to the compulsory courses belonging to the second year of the Medicine grade. The hypothesis states that those courses that use a multiple choice evaluation procedure and verbose slides will be more recall-oriented than those using other evaluation procedures.

Findings: Results show that courses that are more oriented to recall make a larger use of PowerPoint technology, predominating verbal slides, and students have a poorer academic achievement and a lower self-perception of learning.

Limitations: Further studies should encompass a wider sample of courses, years and grades.

Originality/value: Hitherto no study has considered simultaneously the incidence of learning self-perception, evaluation procedures and the use of PowerPoint technology.

Keywords: Assessment procedures, Understanding, Recall, Teaching technology

Jel Codes: I12 


\section{Introducción}

Los planteamientos educativos contemporáneos han prestado una especial atención a la aplicabilidad de los aprendizajes, particularmente orientándolos a la profesionalización (Fernández \& Torres, 2015). Este énfasis ha sido motivado, en parte, por los avances acumulados en los procesos cognitivos vinculados al aprendizaje y, por otra parte, en la constatación de la escasa utilidad del aprendizaje memorístico, de larga tradición académica (Morán, 2010; Pozo, 1999). El cambio fundamental desplaza lo que tradicionalmente era el tipo de aprendizaje objeto ("saber" que se identificaba fundamentalmente con recordar) por un aprendizaje orientado a la comprensión, de la cual se deriva las posibilidades de transformar, ajustar a contextos reales y aplicar el conocimiento (Le Boterf, 2001). Así, el EEES (Espacio Europeo de Educación Superior) centra su interés en el estudiante y en su proceso de aprendizaje, aproximándose al mismo a partir de planteamientos como buscar, además de los conocimientos, la adquisición de competencias académico-profesionales que se evalúan de manera complementaria a los conocimientos específicos de la materia. En la misma línea, el Departamento Australiano de Educación científica y formación, en el año 2002, resalta la importancia de que los estudiantes al finalizar sus estudios hayan adquirido habilidades profesionales, las cuales no pueden ser asumidas a través de la memorización o no entendiendo aquello que se pretende que sea aprendido (Herrero, 2014; Vardi \& Ciccarelli, 2008).

El aprendizaje basado en el recuerdo incluye, además, un conjunto de suposiciones entre las que destacan las tres siguientes: que una vez la información ha accedido a la memoria (cosa que se demuestra al ser recordada) se despliega la comprensión de la misma (Alonso-Tapia, 2002); que la manera en que queda fijado el conocimiento es idéntica para todas las personas; o que el proceso de aprendizaje es esencialmente mecánico (repetición) y se lleva a cabo por parte del alumno durante el estudio. El tipo de acción docente asociada a estos planteamientos consiste en presentar las informaciones a aprender en el formato en el que se esperan que queden fijadas, por lo que el papel del docente es fundamentalmente de transmisor (Cladellas \& Castelló, 2010). Y la forma de evaluar este aprendizaje se basa principalmente en pruebas de recuerdo, en las cuales se comparan los distintos alumnos bajo condiciones estrictamente idénticas, dada la presunción de identidad del aprendizaje (Alfageme-González, Miralles \& Monteagudo, 2015).

En cambio, cuando el tipo de aprendizaje objeto se orienta a la comprensión, los supuestos son claramente distintos: el proceso de aprendizaje es individual, usándose los recursos representacionales y conocimientos previos de cada persona; el conocimiento resultante es también individual, pudiendo tener distintos formatos y organizaciones según la persona; y los mecanismos que conducen a la 
comprensión no son de carácter mecánico sino que implican resolver dudas y reflexionar. Por estas razones, se suele describir al alumno como un agente "activo" en el proceso de aprendizaje (Braxton, Milem \& Sullivan, 2000; Fernández \& Torres, 2015; Huber, 2008; Saiz \& Roman, 2011) ya que dicho aprendizaje es consecuencia de la actividad de cada persona representando y estableciendo conexiones entre contenidos, en lugar de actuar mecánicamente hasta que los contenidos se depositan en la memoria (Alfageme-González et al., 2015; Castelló \& Cladellas, 2013). Dado que el proceso de aprendizaje y el conocimiento resultante son individuales, el papel del docente deja de tener sentido como mero transmisor, del mismo modo que el formato de las informaciones con las que contacta el alumno no tiene por qué coincidir con el conocimiento final, una vez hayan sido elaboradas (Palacios \& López-Pastor, 2013). En efecto, la actividad docente se desplaza de la transmisión al filtrado, la anticipación de dificultades, la planificación de actividades que hagan emerger dudas o detectar contradicciones en el alumnado y, de manera general, a la provisión de guía y feedback al proceso individual de aprendizaje (Hartell, Gumaelius \& Svärdh, 2015). La Guía de Educación Superior de la Comunidad Europea (EC, 2001) anima a los profesores a cambiar su rol, de manera que la concepción clásica de transmisor de conocimientos sea sustituida por la de guía o facilitador de aprendizaje. De manera semejante, la evaluación basada en el recuerdo pierde su interés y, además, el objeto de la misma ya no es el de comparar alumnos que se supone han realizado un mismo proceso, sino detectar posibles errores o faltas de completitud a fin de optimizar dicho aprendizaje (Carless, Joughin \& Mok, 2006; Padilla-Carmona, Gil-Flores, Rodríguez-Santero, Torres-Gordillo \& Clares-López, 2010).

Dada la prolongada prevalencia del modelo de aprendizaje basado en el recuerdo, las directrices de los planteamientos contemporáneos, orientadas a la comprensión y la aplicación, se consolidan de manera lenta, existiendo todavía numerosas acciones educativas orientadas exclusiva o mayoritariamente al recuerdo (Biggs, 2005; De Miguel, 2006; Ruiz-Gallardo, Castaño, Gómez-Alday \& Valdés, 2010). A modo de ejemplo, un estudio desarrollado en España muestra que el 60\% de los profesores siempre o casi siempre suele impartir su docencia a través de clases magistrales (Baena, González, López, Riera, Martel \& Pacheco, 2004). En general, cuando los docentes asumen - de manera explícita o implícita este planteamiento, adoptan el rol de transmisor de información y presentan un cuerpo de contenidos en el formato que esperan que sea recordado por el alumnado (Breton, 1999; Vardi \& Ciccarelli, 2008). Complementariamente, en la forma de evaluación empleada suele primar la comparabilidad y se orienta básicamente al recuerdo o elaboraciones poco complejas de los contenidos (Azer, 2009) para lo cual las pruebas "tipo test" son ideales. Por el contrario, en los casos en que la actividad docente se orienta hacia la comprensión, la información aportada por los docentes es bastante menos fija, ya que su función no es la de transmitir el producto final, sino destacar elementos fundamentales y propiciar 
oportunidades para que cada alumno elabore y reflexione sobre los contenidos, en el tránsito hacia la construcción de su conocimiento (Dweyer, Hogan \& Stewart, 2010). De manera afín, el modelo de evaluación que era coherente con los planteamientos orientados al recuerdo deja de serlo en estos casos, substituyéndose por procedimientos que permitan exponer aprendizajes de mayor complejidad (estructuras que incluyan diversos elementos, interacciones entre los mismos, aplicaciones, portafolios, etcétera) (Morán, 2010). Aquí, las pruebas más ajustadas son las que permiten una cierta elaboración discursiva (sean orales o escritas) o la solución de casos y problemas de cierta complejidad (Veeravagu, Muthusamy, Marimuthu \& Michael, 2010).

La evaluación tiene un alto impacto en el aprendizaje de los estudiantes (Heeneman, Oudkerk, Schuwirth, Van der Vleuten \& Driessen, 2015), por lo que ello conjuntamente con las ideas mencionadas hasta aquí sugieren que la forma de evaluación predominante en una determinada asignatura pueda ser considerada como una prueba del tipo de aprendizaje objeto que se espera conseguir en la misma, por lo que se puede razonablemente esperar que el planteamiento global de la docencia cumpla de manera coherente con el resto de condiciones antes descritas. El uso de las TIC en general (Mackiewicz, Mastarone \& Lee-Kim, 2006) y del muy extendido recurso del PowerPoint en particular (Mackiewicz, 2008) también se articula desde este mismo marco: si el enfoque se orienta al recuerdo, los contenidos presentados tecnológicamente probablemente se ajusten al formato final a ser recordado, mientras que cuando la orientación es hacia la comprensión, la tecnología será usada para ilustrar situaciones, mostrar interacciones o síntesis de elementos que puedan facilitar el aprendizaje individual (Cladellas, Castelló, Badia \& Cirera, 2013). Hertz, Woerkum y Kerkhof, (2015) plantean que el uso del PowerPoint por parte de muchos docentes, de hecho, se orienta a sustituir su función docente más que a complementarla. Por tanto, las slides expositivas son un producto muy abundante en la práctica docente en general y, en particular, universitaria, aunque ello no implica que comporte ventaja instruccional alguna.

Otros autores (Ankad, Shashikala, Herur, Manjula, Chinagudi \& Patil, 2015) han analizado los diferentes estilos de aprendizaje de los alumnos, así como las diferencias de género en relación con el aprovechamiento obtenido después de sesiones de PowerPoint en diferentes formatos. Sus resultados ponen de manifiesto que ni los estilos de aprendizaje ni el género tienen interacción alguna con el aprendizaje experimentado. Por lo tanto, las características individuales del alumnado no parecen ser una característica relevante a la hora de explicar los efectos del tipo de tecnología empleada. 
También resulta razonable esperar que el procedimiento de evaluación determine de qué manera el alumnado abordará una determinada asignatura: desde la toma de apuntes hasta los procesos cognitivos implicados están condicionados por lo que se espera que tenga transcendencia en el resultado final.

Veeravagu et al. (2010) plantean el uso de las tradicionales taxonomías de Bloom tanto para describir el aprendizaje como para establecer el tipo de evaluación más pertinente. Así, cuando se consigue cierta comprensión de los contenidos suele reflejarse en una mayor satisfacción por la asignatura y la percepción de haber aprendido. Por contra, la estricta memorización, por tratarse de un proceso mecánico, es mucho menos satisfactoria, del mismo modo que la percepción de utilidad del aprendizaje es bastante más vaga. Finalmente debe considerarse que, mientras la memorización no conduce a la comprensión, el aprendizaje comprensivo sí que permite el recuerdo, al menos de los aspectos relevantes de los contenidos. En conjunto, pues, los planteamientos orientados a la comprensión permiten esperar una mayor satisfacción por el proceso de aprendizaje, que bien puede considerarse una forma de motivación (Cannon \& Newble, 2000; Hwang, Kuo, Chen \& Ho, 2014), del mismo modo que no tienen por qué conducir a un rendimiento menor, incluso en términos de recuerdo (Savoy, Proctor \& Salvendy, 2009).

A tenor de los argumentos expuestos hasta este punto se plantean las siguientes hipótesis: (H1) "Se espera encontrar una relación positiva entre la evaluación mediante pruebas tipo test y el uso de transparencias de tipo verbal (textos o esquemas)". Esta hipótesis se deriva del hecho que los planteamientos docentes orientados a la transmisión de informaciones, tienden a representar estas informaciones de manera estable, razón por la cual pueden tomar forma de diapositivas que fijen estos contenidos, los cuales se espera que sean representados por el alumnado literalmente en este mismo formato. En cambio, los planteamientos docentes orientados a la comprensión, suelen asumir que es cada alumno quien llevará a cabo una elaboración de los contenidos y una expresión final de los mismos en sus propias palabras. No es que no puedan establecer definiciones precisas, sino que una parte considerable de los contenidos tiene carácter de ilustración, de aplicación o de razonamiento más que de enunciación. Y (H2) "Existe una relación inversa entre la utilización de esta tecnología, con contenidos predominantemente verbales, y la percepción de aprendizaje y el rendimiento del alumno". Esta hipótesis se refiere a que el primer planteamiento comporta un papel pasivo por parte del alumno, ya que solamente debe introducir en su memoria un material preparado externamente que no puede modificar o elaborar. Pero esta elaboración personal de los contenidos es la que se encuentra más directamente relacionada con la comprensión, del mismo modo que la aplicación de los contenidos en problemas o casos consolida la percepción de dominio de los mismos. Consecuentemente, es de 
esperar que la percepción de aprendizaje y la valoración de las materias sean significativamente menores en el primer tipo de asignaturas.

\section{Método}

\subsection{Participantes}

Del total de alumnos de primero de Medicina de la Universidad Autónoma de Barcelona se realizó un muestreo estratificado aleatorio con el fin de seleccionar la muestra. La muestra definitiva quedó constituida por un total de 63 participantes. De cada participante se obtuvieron cinco registros correspondientes a cada una de las cinco asignaturas obligatorias del plan de estudios del primer semestre de primero de Medicina. Así, pues al final se obtuvieron un total de 315 registros.

De la muestra inicial de estudiantes (101) fueron descartados un total de 38, 18 de ellos por no haber cursado alguna de las cinco asignaturas anteriormente señaladas, y los 20 restantes por no acudir a la cita de obtención de datos o por no presentar el expediente académico del curso anterior, que era una de las variables evaluadas. Todos los participantes actuaron de forma voluntaria y sin recibir ningún tipo de contraprestación por ello.

\subsection{Instrumentos}

Se elaboró un cuestionario para recoger información acerca de cada una de las cinco asignaturas evaluadas así como de la percepción de aprendizaje por parte del alumno. Las preguntas hacían referencia al porcentaje de uso del PowerPoint en general y también desglosado en los siguientes tipos: visual, diagrama o verbal, al tipo de evaluación utilizado por el profesor y a la valoración del estudiante respecto a lo aprendido en la asignatura. Una versión parcial de este cuestionario se había puesto a punto en una fase piloto en la cual los alumnos matriculados en las asignaturas de los autores, respondían al uso de tecnología y el tipo de evaluación. Los resultados mostraron que la diferencia entre la media de las estimaciones de los alumnos y la frecuencia real de uso de cada tipo de transparencias no superaba nunca el 5\% y que en el tipo de evaluación las diferencias se redujeron a menos de un 3\%. Dado que la pregunta añadida para el presente estudio se correspondía con la percepción del alumno de su propio aprendizaje, no había lugar para utilizar ningún procedimiento de validación de la misma. 


\subsection{Variables}

Las variables analizadas en el presente estudio son:

- Variable independiente:

- Tipo de evaluación. Las categorías fueron:

- sólo se empleaban exámenes tipo test,

- otro tipo de exámenes (oral o escrito de preguntas abiertas) o bien

- un tipo de evaluación mixto (examen tipo test combinado con trabajos de índole diversa, con la condición de que los exámenes tipo test no superaran el 50\%).

- Variables dependientes:

- Porcentaje de clases de cada asignatura en las que se empleó el PowerPoint.

- Porcentaje de tipos de PowerPoint. En concreto, se medía su uso para la presentación de los contenidos en forma visual (gráficos e imágenes), diagrama (combinación de gráficos y contenido verbal) y verbal (textos y esquemas).

- Valoración del aprendizaje. Se pidió a cada alumno que valorara la cantidad de aprendizaje que había realizado en cada asignatura, en una escala de 0 a 10. Sin tratarse de una medida objetiva del aprendizaje efectivo, esta variable incluye una aproximación al mismo, probablemente combinada con el interés despertado por la asignatura.

- Nota obtenida. Nota numérica obtenida en cada una de las cinco asignaturas, con un decimal y valores entre 0 y 10. La fuente de esta información era el expediente académico.

Las diferencias en cuanto a las características de asignaturas y contenidos no eran suficientes para anular la toma de decisiones docentes basada en el enfoque del aprendizaje objeto adoptado por cada profesor. 


\subsection{Procedimiento}

Los investigadores se encargaron de contactar con las personas seleccionadas y concretar día y hora para que respondieran al cuestionario. La cumplimentación del mismo se realizó en el laboratorio ante la presencia de uno de los autores de este trabajo y en un tiempo medio de 15 minutos.

Durante la misma sesión, y a petición previa, se les instaba a presentar una copia de la calificación obtenida en las asignaturas cursadas durante el primer semestre con el objetivo de verificar la nota obtenida en cada una de las asignaturas seleccionadas para el estudio.

El procedimiento de recogida de datos se llevó a cabo durante el mes de marzo con el propósito de que hubiera transcurrido el mínimo tiempo desde la finalización del semestre, y así minimizar los efectos de memoria.

\subsection{Análisis de datos}

Tomando el tipo de evaluación empleada como variable independiente, las respuestas fueron sometidas a un análisis de varianza "ANOVA" de un factor, para poner a prueba si los diferentes tipos de evaluación diferían de manera estadísticamente significativa en las variables dependientes (\% uso PowerPoint, \% ponderado de PowerPoint de tipo visual, \% ponderado de PowerPoint de tipo de diagrama y \% ponderado de PowerPoint de tipo verbal, Nota obtenida, valoración del aprendizaje obtenido). La ponderación en los tipos de PowerPoint consistió en multiplicar el porcentaje relativo de cada tipo de transparencia por el porcentaje total de uso del PowerPoint en cada asignatura, de manera que se obtenía la proporción de sesiones en que se utilizó cada tipo. Se obtuvo el valor Eta al cuadrado parcial ( $\eta$ p2) como medida del tamaño del efecto, siguiendo el criterio de Huberty (2002) que considera que una Eta al cuadrado parcial de .01 es pequeña, .04 moderada, y .1 grande. Además de los contrastes post hoc con el método de Scheffé, se aproximó la significación de los mismos mediante la prueba de comparación de medias basada en el índice t de Student. Se realizó también un análisis de correlación entre las variables dependientes, mediante el algoritmo de Pearson con significación bilateral. Estas correlaciones se realizaron a fin de tener una estimación de la varianza compartida por las variables dependientes.

Los cálculos se realizaron con el paquete estadístico SPSS/PC+ (versión 15.0; Chicago, IL, USA) y los tests estadísticos fueron bilaterales con error tipo I variable al 5\%. 


\section{Resultados}

La distribución de los registros por tipo de evaluación fue de $23.49 \%$ en examen sólo tipo test, 30.48\% en otros exámenes y $46.03 \%$ en evaluación mixta.

\subsection{Contrastes entre grupos}

La Tabla 1 muestra el total de medias, error típico, F-test y potencia observada para cada una de las variables dependientes en las tres condiciones de evaluación.

\begin{tabular}{|c|c|c|c|c|c|}
\hline \multirow[b]{2}{*}{$\begin{array}{c}\text { VARIABLES } \\
\text { DEPENDIENTES }\end{array}$} & \multicolumn{3}{|c|}{ TIPO DE EVALUACIÓN } & \multirow[b]{2}{*}{$\mathbf{F}$} & \multirow[b]{2}{*}{$\begin{array}{c}\text { Potencia } \\
\text { observada }\end{array}$} \\
\hline & $\begin{array}{c}\text { Examen tipo } \\
\text { test }(n=74)\end{array}$ & $\begin{array}{l}\text { Otros exámenes } \\
(\mathrm{n}=96)\end{array}$ & $\begin{array}{l}\text { Evaluación mixta } \\
\qquad(\mathrm{n}=145)\end{array}$ & & \\
\hline$\%$ uso PowerPoint & $83.93(2.23)$ & $64.88(3.47)$ & $73.45(2.28)$ & $9.338^{* *}$ & .990 \\
\hline $\begin{array}{l}\% \text { ponderado PowerPoint de } \\
\text { tipo visual }\end{array}$ & $16.73(1.97)$ & $12.92(1.87)$ & $13.01(1.46)$ & 1.258 & .206 \\
\hline $\begin{array}{l}\% \text { ponderado PowerPoint de } \\
\text { tipo diagrama }\end{array}$ & $15.89(1.96)$ & $17.24(2.08)$ & $18.99(1.60)$ & .679 & .141 \\
\hline $\begin{array}{l}\% \text { ponderado PowerPoint de } \\
\text { tipo verbal }\end{array}$ & $51.30(3.34)$ & $34.13(3.04)$ & $41.31(2.28)$ & $7.511^{*}$ & .950 \\
\hline Nota obtenida & $6.79(.20)$ & $7.55(.13)$ & $7.50(.11)$ & $7.660^{*}$ & .967 \\
\hline Valoración del aprendizaje & $6.14(.21)$ & $7.04(.20)$ & $6.91(.17)$ & $4.940^{*}$ & .749 \\
\hline
\end{tabular}

Tabla 1. Medias y error típico (entre paréntesis) de las variables dependientes obtenidas en las tres situaciones experimentales

Se manifestaron diferencias significativas $(\mathrm{F}(2,302)=9.338 ; \mathrm{p}<.001 ; \eta \mathrm{p} 2=.068)$. En concreto, existe una mayor utilización en los casos de examen tipo test, en comparación con otros exámenes $(\mathrm{p}<.001)$ y evaluación mixta $(\mathrm{p}=.038)$.

\section{2. \% ponderado PowerPoint de tipo visual y \% ponderado PowerPoint de tipo diagrama}

No se observaron diferencias significativas entre los tres grupos para cada una de las variables.

\section{3. \% ponderado PowerPoint de tipo verbal}

Se manifiestan diferencias significativas $\mathrm{F}(2,302)=7.511 ; \mathrm{p}<.05 ; \eta \mathrm{p} 2=.050)$. Este formato se usa predominantemente en los casos de examen tipo test, a diferencia de otros exámenes ( $\mathrm{p}=.001)$. 


\subsection{Nota obtenida}

Se observan diferencias significativas $\mathrm{F}(2,302)=7.660 ; \mathrm{p}<.001 ; \eta \mathrm{p} 2=.055)$. La nota es menor en la evaluación de examen tipo test comparada con otros exámenes $(\mathrm{p}=.002)$ y con evaluación mixta $(\mathrm{p}=.002)$.

\subsection{Valoración del aprendizaje}

Se observan diferencias significativas $\mathrm{F}(2,302)=4.940 ; \mathrm{p}<.001 ; \eta \mathrm{p} 2=.028)$. La valoración es menor en el caso de examen tipo test en comparación con otros exámenes $(\mathrm{p}=.014)$ y con evaluación mixta $(\mathrm{p}=.026)$.

\subsection{Correlaciones entre variables dependientes}

La Tabla 2 muestra las correlaciones entre todas las variables estudiadas. El \% uso PowerPoint correlaciona significativamente con todas las variables; de forma positiva con las variables relacionadas con el tipo de uso del PowerPoint, y de forma negativa con las variables nota obtenida y valoración de la asignatura. El tipo de PowerPoint verbal correlaciona negativamente y de forma significativa con el tipo de PowerPoint visual y diagrama. La nota obtenida correlaciona negativamente y de forma significativa cuando el tipo de PowerPoint es verbal, y por último, se observa una alta correlación significativamente positiva entre la nota obtenida y la valoración de la asignatura efectuada por los estudiantes.

\begin{tabular}{|l|l|r|r|r|r|}
\cline { 2 - 6 } & \% uso PowerPoint & $\begin{array}{c}\text { \% ponderado } \\
\text { PowerPoint } \\
\text { visual }\end{array}$ & $\begin{array}{c}\text { \% } \\
\text { ponderado } \\
\text { PowerPoint } \\
\text { diagrama }\end{array}$ & $\begin{array}{c}\text { \% } \\
\text { ponderado } \\
\text { PowerPoint } \\
\text { verbal }\end{array}$ & $\begin{array}{c}\text { Nota } \\
\text { obtenida }\end{array}$ \\
\hline $\begin{array}{l}\text { \% ponderado } \\
\text { PowerPoint visual }\end{array}$ & $.265^{* *}$ & & & & \\
\hline $\begin{array}{l}\text { \% ponderado } \\
\text { PowerPoint diagrama }\end{array}$ & $.381^{* *}$ & -.001 & & & \\
\hline $\begin{array}{l}\text { \% ponderado } \\
\text { PowerPoint verbal }\end{array}$ & $.603^{* *}$ & $-.341^{* *}$ & $-.271^{* *}$ & & \\
\hline Nota obtenida & $-.254^{* *}$ & -.018 & -.065 & $-.204^{* *}$ & \\
\hline Valoración asignatura & $-.181^{* *}$ & -.038 & -.095 & -.095 & $.606^{* *}$ \\
\hline
\end{tabular}

$* * \mathrm{p}<.001$

Tabla 2. Correlaciones entre las variables dependientes 
Las dos correlaciones más elevadas se observan entre Uso total de PowerPoint y la tipología verbal del mismo, así como entre valoración de la asignatura y nota obtenida. Teniendo en cuenta que cualquiera de ellas pone en evidencia que la varianza compartida por el par de variables es superior al $36 \%$, parecen delatar una sólida asociación de las transparencias verbales con la utilización de esta TIC, del mismo modo que entre la percepción de aprendizaje y el rendimiento en la asignatura.

\section{Discusión y conclusiones}

Los resultados corroboran que un mayor uso del PowerPoint y, en concreto, el uso de slides con formato verbal, tiene una relación positiva con el tipo de evaluación empleado (examen tipo test). Este resultado es acorde con la primera hipótesis planteada y pondría de manifiesto la existencia de un enfoque del proceso educativo mayoritariamente orientado al recuerdo en esas asignaturas. Asimismo, los resultados muestran como la presentación de los contenidos en otros formatos (diagrama o visual) no tienen relación con la implantación de un determinado tipo de evaluación.

En la línea de lo planteado por Cladellas et al. (2013) se manifiesta que, más que el uso del PowerPoint en la impartición de clases, es pertinente hablar del formato de los contenidos presentados. Las imágenes o gráficos pueden redundar en un buen aprendizaje, mientras que la abundancia de transparencias con contenidos verbales puede ser contraproducente (Savoy et al., 2009). El efecto contraproducente de las diapositivas con formato verbal es acorde con los resultados obtenidos por Amare (2006), Cladellas et al. (2013) y Erwin y Rieppi (1999). Estos autores argumentan que, ante este tipo de diapositivas, el estudiante está más pendiente del material presentado que del discurso del docente, o, en el mejor de los casos, debe repartir su atención entre uno y otro. Pero lo más habitual, como indican Driessnack (2005) y Savoy et al. (2009), es que se centre en la copia del material presentado, actuando la información verbal que pueda facilitar el docente como estímulo distractor (Szabo \& Hastings, 2000). Cuando el profesor se limita a presentar (o leer) los contenidos verbales de las diapositivas, no ejerce ninguna función facilitadora del aprendizaje, ya que interactúa con el material proyectado y no con los alumnos (Cladellas \& Castelló, 2010).

Los resultados también muestran que las notas obtenidas a partir de aquellos sistemas de evaluación orientados a la comprensión son mejores que los de las asignaturas con evaluaciones orientadas al recuerdo. Aunando este dato con la asociación del mayor uso de PowerPoint verbal en este último grupo de asignaturas, se podría afirmar que dicho formato de PowerPoint no contribuye a un mejor rendimiento académico. 
Cabe destacar que las notas obtenidas en los casos de evaluaciones mixtas, que incluían una prueba tipo test junto con otras, no han sido significativamente inferiores a las de las asignaturas sin prueba tipo test. Este resultado pone de manifiesto que un proceso de aprendizaje orientado a la comprensión también da soporte al tipo de recuerdo evaluado por las pruebas tipo test.

Las asignaturas que han generado una mayor percepción de aprendizaje han sido las que no utilizaban pruebas tipo test $y$, tal como se ha indicado, menores frecuencias de presentaciones PowerPoint de carácter verbal así como un menor uso de esta TIC en general. Este dato es congruente con la segunda hipótesis, consolidando la idea de que los planteamientos de aprendizaje en los que el alumno se supone desempeña un papel más activo no sólo redundan en una mayor motivación, sino también en un mejor rendimiento, como acreditan las correlaciones que muestran la intensa relación entre estas dos variables.

El presente trabajo tiene como principal aportación la integración coherente de la manera en que los docentes representan el aprendizaje con el procedimiento de evaluación del mismo y el aprendizaje finalmente obtenido por los alumnos. Estas tres variables, tratadas de manera conjunta, no habían sido abordadas hasta ahora. De manera secundaria, el resultado que muestra que los alumnos no sólo perciben mayor aprendizaje sino que obtienen un mejor rendimiento cuando se permite que sean quienes elaboran la información, aunque no es novedoso (Castelló \& Cladellas, 2013), supone una clara referencia a que el foco del proceso de enseñanza debe orientarse claramente a quien realiza el aprendizaje, más que a los contenidos impartidos. Y, en tercer lugar, insistir en que la tecnología puede resultar una ayuda en este proceso pero no en cualquier formato. No se trata tanto de que las slides verbales sean intrínsecamente negativas, sino que su uso interfiere con otro canal verbal (el discurso docente, al que añade poco o nada) y su predominio pone de manifiesto un enfoque docente basado en los contenidos y no en el alumno (Brandl, Schneid \& Armour, 2015).

Este trabajo no está exento de limitaciones: la alta exclusión inicial de participantes (37.6\%) por no haber cursado alguna de las asignaturas o no haber aportado la documentación acreditativa de las notas podría actuar como elemento distorsionador de los resultados obtenidos. Próximos estudios deberían contemplar muestras más grandes de asignaturas que abarcara un mayor número de cursos académicos y no centrarse sólo en un único curso. Por otro lado, sería conveniente realizar una evaluación formal del aprendizaje efectivo de los alumnos y no quedarse solo con la percepción que tienen estos sobre el aprendizaje alcanzado. Para ello sería necesario realizar algún tipo de evaluación de los contenidos de la asignatura que perduran un tiempo después de haberla realizado. Esto aportaría datos objetivos, evitando las distorsiones de la valoración del aprendizaje por parte del propio alumno. 


\section{Referencias}

Alfageme-González, M.B., Miralles, P., \& Monteagudo, J. (2015). Cómo evalúa el profesorado de Geografía e Historia de Enseñanza Secundaria. Revista Complutense de Educación, 26(3), 571-589. https://doi.org/10.5209/rev_RCED.2015.v26.n3.44428

Alonso-Tapia, J. (2002). Knowledge assessment and conceptual understanding. En: M. Limón \& L. Mason (Eds.), Reframing the processes of conceptual change (pp. 389-413). Dordrecht: Kluwer. https://doi.org/10.1007/0-306-47637-1_19

Amare, N. (2006). To slideware or not to slideware: Students' experiences with PowerPoint vs. Lecture. Journal of Technical Writing and Communication, 36(3), 297-308. https://doi.org/10.2190/03GX-F1HW-VW5M7DAR

Ankad, R.B., Shashikala, G.V., Herur, A., Manjula, R., Chinagudi, S., \& Patil, S. (2015). PowerPoint presentation in learning physiology by undergraduates with different learning styles. Advances in Physiology Education, 39(1), 367-371. https://doi.org/10.1152/advan.00119.2015

Azer, S.A. (2009). What makes a great lecture? Use of lectures in a hybrid PBL curriculum. The Kaobsiung Journal of Medical Sciences, 25(3), 109-115. https://doi.org/10.1016/S1607-551X(09)70049-X

Baena, M.D., González, M.C., López, P., Riera, C., Martel, M.C., \& Pacheco, M.A. (2004). El ECTS y una Universidad que aprende. Revista Electrónica Interuniversitaria de Formación del Profesorado, 18, 8(3), $1-5$.

Biggs, J. (2005). Calidad del aprendizaje universitario. Madrid: Narcea.

Brandl, K., Schneid, S., \& Armour, C. (2015). Writing on the Board versus Powerpoint: What do Students Prefer and Why?. The FASEB Journal, 29(1 Supplement), LB465.

Braxton, J.M., Milem, J.F., \& Sullivan, A.S. (2000). The influence of Active Learning on the College Student Departure Process. Journal of Higher Education, 71(5), 569-590. https://doi.org/10.2307/2649260

Breton, G. (1999). Some empirical evidence on the superiority of the problem-based learning (PBL) method. Accounting Education, 8(1), 1-12. https://doi.org/10.1080/096392899331008

Cannon, R., \& Newble, D. (2000). A Handbook for teachers in Universities and Colleges. A guide to improving Teaching Methods. London: Kogan Page.

Carless, D., Joughin, G., \& Mok, M. (2006). Learning-oriented assessment: Principles and practice. Assessment \& Evaluation in Higher Education, 31(4), 395-398. https://doi.org/10.1080/02602930600679043 
Castelló, A., \& Cladellas, R. (2013). La evaluación de la comprensión en el aprendizaje: El empleo de las TIC en el análisis de estructuras de conocimiento. Estudios Pedagógicos, 39, 41-57. https://doi.org/10.4067/S0718-07052013000300004

Cladellas, R., \& Castelló, A. (2010). Aportes y perjuicios de las TIC’s a la educación. Congreso de Alfabetización mediática y culturas digitales. Sevilla.

Cladellas, R., Castelló, A., Badia, M., \& Cirera, M. (2013). Effects of the PowerPoint methodology on content learning. Intangible Capital, 9(1), 184-198.

De Miguel, M. (2006). Métodos y modalidades de enseñanza en la educación superior. En M. De Miguel (Ed.), Metodologías de enseñanza y aprendizaje para el desarrollo de competencias (pp. 17-52). Madrid: Alianza editorial.

Driessnack, M. (2005). A closer look at PowerPoint Feature Article. Journal of Nursing Education, 44(8), 347.

Dweyer, C.P., Hogan, M.J., \& Stewart, I. (2010). The evaluation of argument mapping as a learning tool: Comparing the effects of map reading versus text reading on comprehension and recall of arguments. Thinking Skills and Creativity, 5(1), 16-22. https://doi.org/10.1016/j.tsc.2009.05.001

EC (European Commission). (2001). Hacer realidad un espacio europeo del aprendizaje permanente. Comisión Europea. Dirección General de Educación y Cultura. COM (2001) 678 Final. Bruselas, 21.11.2001. Disponible online en: http://www.ub.es/ub/europa/documents2/2 Comunicats de la Comissio/06 COM Aptge permanent.pdf (Fecha del último acceso: 20 de Diciembre de 2005).

Erwin, T.D., \& Rieppi, R. (1999). Comparing multimedia and traditional approaches in undergraduate psychology classes. Teaching of Psychology, 26(1), 58-61. https://doi.org/10.1207/s15328023top2601_18

Fernández, J.M., \& Torres, J.A. (2015). Actitudes docentes y buenas prácticas con TIC del profesorado de Educación Permanente de Adultos en Andalucia. Revista Complutense de Educación, 26, 33-49.

Hartell, E., Gumaelius, L., \& Svärdh, J. (2015). Investigating technology teachers' self-efficacy on assessment. International Journal of Technology and Design Education, 25, 321-337. https://doi.org/10.1007/s10798-014-9285-9

Hertz, B., Woerkum, C., \& Kerkhof, P. (2015). Why do scholars use PowerPoint the way they do? Business Communication Quarterly, 78(3), 273-291. https://doi.org/10.1177/2329490615589171 
Hwang, G.J., Kuo, F.R., Chen, N.S., \& Ho, H.J. (2014). Effects of an integrated concept mapping and web-based problem-solving approach on students' learning achievements, perceptions and cognitive loads. Computers \& Education, 71, 77-86. https://doi.org/10.1016/j.compedu.2013.09.013

Heeneman, S., Oudkerk, A., Schuwirth, L.W., Van der Vleuten, C.P., \& Driessen, E.W. (2015). The impact of programmatic assessment on student learning: Theory versus practice. Medical Education, 49, 487-498. https://doi.org/10.1111/medu.12645

Herrero, R.M. (2014). El papel de las TIC en el aula universitaria para la formación en competencias del alumnado. Pixel-Bit. Revista de Medios y Educación, 45, 173-188. https://doi.org/10.12795/pixelbit.2014.i45.12

Huber, G.L. (2008). Aprendizaje activo y metodologías educativas. Revista de Educación, No. Extra, 59-81.

Huberty, C.J. (2002). A history of effect sizes indices. Educational and Psychological Measurement, 67, 227-240. https://doi.org/10.1177/0013164402062002002

Le Boterf, G. (2001). Ingeniería de las competencias. Barcelona: Gedisa.

Mackiewicz, J. (2008). Comparing PowerPoint experts' and university students' opinions about PowerPoint presentations. Journal Technical Writing and Communication, 38(2), 149-165. https://doi.org/10.2190/TW.38.2.d

Mackiewicz, J., Mastarone, G., \& Lee-Kim, J. (2006). What's Not to Like? Business Student's Opinions about PowerPoint Slide Design. Proceedings of the 2006 Association for Business Communication Annual Convention, pp. 1-9.

Morán, P. (2010). Aproximaciones teórico-metodológicas en torno al uso del portafolio como estrategia de evaluación del alumno en la práctica docente: Experiencia en un curso de Laboratorio de Didáctica en la docencia universitaria. Perfiles educativos, 32(129), 102-128.

Padilla-Carmona, M.T., Gil-Flores, J., Rodríguez-Santero, J., Torres-Gordillo, J.J., \& Clares-López, J. (2010). Evaluando el sistema de evaluación del aprendizaje universitario: Análisis documental aplicado al caso de la Universidad de Sevilla. Revista Iberoamericana de Educación, 53(3), 1-14.

Palacios, A., \& López-Pastor, V. (2013). Haz lo que yo digo pero no lo que yo hago: Sistemas de evaluación del alumnado en la formación inicial del profesorado. Revista de Educación, 361, 279-305.

Pozo, I. (1999). Aprender y enseñar ciencia. Madrid: Morata. 
Ruiz-Gallardo, J.R., Castaño, S., Gómez-Alday, J.J., \& Valdés, A. (2010). Assessing student workload in Problem Based Learning: Relationships among teaching method, student workload and achievement. A case study in Natural Sciences. Teaching and Teacher Education, 27(3), 619-627. https://doi.org/10.1016/j.tate.2010.11.001

Saiz, M.C., \& Roman, J.M. (2011). Cuatro formas de evaluación en educación superior gestionadas desde la tutoria. Revista de Psicodidáctica, 16(1), 145-161.

Savoy, A., Proctor, R. W., \& Salvendy, G. (2009). Information retention from PowerPoint and traditional lectura. Computers and Education, 52, 858-867. https://doi.org/10.1016/j.compedu.2008.12.005

Szabo, A., \& Hastings, N. (2000). Using IT in the undergraduate classroom: Should we replace the blackboard with PowerPoint?. Computers \& Education, 35, 175-187. https://doi.org/10.1016/S03601315(00)00030-0

Vardi, I., \& Ciccarelli, M. (2008). Overcoming problems in problem-based learning: A trial of strategies in an undergraduate unit. Innovations in Education and Teaching International, 45, 345-354. https://doi.org/10.1080/14703290802377190

Veeravagu, J., Muthusamy, Ch., Marimuthu, R., \& Michael, A.S. (2010). Using Bloom's Taxonomy to Gauge Students’ Reading Comprehension Performance. Canadian Social Science, 6(3), 205-212.

Intangible Capital, 2017 (www.intangiblecapital.org)

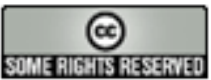

Article's contents are provided on an Attribution-Non Commercial 3.0 Creative commons license. Readers are allowed to copy, distribute and communicate article's contents, provided the author's and Intangible Capital's names are included. It must not be used for commercial purposes. To see the complete license contents, please visit http://creativecommons.org/licenses/by-nc/3.0/. 